Experimentally-Enhanced Perceptions of Meaning Confer Resilience to the Interpersonal Adversity Implicated in Suicide Risk 


\begin{abstract}

\section{Background and Objectives}

The interpersonal theory of suicide proposes that perceived burdensomeness and thwarted belongingness (PB-TB) are proximal causes of suicidal desire. Here we test whether experimentally induced perceptions of meaningfulness can confer resilience against interpersonal adversity and enhance persistence, the erosion of which is a potential antecedent to suicide risk.
\end{abstract}

\title{
Methods
}

Undergraduate university students $(N=93)$ were randomly allocated to complete a team task under conditions of high or low task-extrinsic meaning and high or low PB-TB. Participants in the high task-extrinsic meaning condition were given the opportunity to donate to a charity as part of their experimental participation, whereas those in the low task-extrinsic meaning condition were not.

\section{Results}

Consistent with the buffering hypothesis that suicide resilience is active only when adversity is heightened, participants in the high task-extrinsic meaning condition who reported higher levels of perceived meaningfulness displayed greater willingness to persist in the face of experimentally-induced high PB and TB compared to those in the low task-extrinsic meaning condition and those in conditions where the interpersonal adversity was not induced (low PB and TB).

\section{Limitations}

The meaning induction was effective only in a subset of participants. The dual induction of PB and TB also precludes inferences about their independent causal effects on willingness to persist.

\section{Conclusions}


Meaning-making interventions may attenuate the impact of proximal interpersonal antecedents of suicidal desire. Enhancing resilience in this manner can potentially improve the efficacy of prevention efforts beyond the direct amelioration of suicide risk factors.

Keywords: interpersonal theory of suicide, experimental psychopathology, meaning and purpose, life-sustaining factors, suicide resilience 


\section{Introduction}

The interpersonal theory of suicide (ITS; Joiner, 2005) proposes that a desire for suicide arises when two fundamental human needs are thwarted: the need to contribute and the need to belong. These proximal causal factors manifest as perceptions that one is a burden on others (perceived burdensomeness) and that one is lacking meaningful interpersonal connections (thwarted belongingness). An individual experiencing heightened levels of these aversive states views suicide as a means of escape from this interpersonal adversity. The notion of suicide as an escape from psychological pain is reflected in several contemporary theories (e.g., Baumeister, 1990; O’Connor, Platt, \& Gordon, 2011) and highlights the importance of persistence in adversity. The current study uses an experimental paradigm (Collins, Best, Stritzke, \& Page, 2016) to test the role of perceived meaningfulness as a life-sustaining factor that may increase persistence and reduce one's desire to escape in the face of experimentally-induced perceived burdensomeness and thwarted belongingness.

Perceived burdensomeness and thwarted belongingness are associated with increased risk for suicidal desire in diverse samples including adolescents (Barzilay et al., 2015), young adults (Joiner et al., 2009), psychotherapy outpatients (Van Orden, Lynam, Hollar, \& Joiner, 2006), and community cohorts (Christensen, Batterham, Soubelet, \& Mackinnon, 2013). Recent experimental evidence also supports the causal effects of these constructs on desire to escape as a potential antecedent to suicide risk and highlights the importance of resilience factors that may protect against suicidality even when interpersonal adversity is heightened (Collins et al., 2016; George, Collins, Cao, Stritzke, \& Page, 2017). Factors conferring resilience are posited to exist on a separate dimension to risk and serve to mitigate the impact of risk factors on subsequent suicidality (Cheavens, Cukrowicz, Hansen, \& Mitchell, 2016; Johnson, Wood, Gooding, Taylor, 
\& Tarrier, 2011). Identifying modifiable resilience factors may therefore enhance the effectiveness of suicide prevention efforts by providing targets for intervention that complement the direct amelioration of suicidal ideation and its interpersonal antecedents (Collins, Stritzke, Page, Brown, \& Wylde, 2018).

The perception that life has meaning and is therefore worth sustaining is arguably a key factor underlying persistence in adversity (Park, 2010). Conversely, a thwarting of the pursuit of meaning can lead to apathy, hopelessness, and diminished motivation (Baumeister, Vohs, Aaker, \& Garbinsky, 2013; Frankl, 1959; Kashdan \& McKnight, 2009; Ryan \& Deci, 2000). One pathway to meaningfulness involves possessing goals that transcend self-interest and current circumstances (Reker, 2000). These goals form part of a framework that facilitates understanding how life experiences, including adverse events, are part of a greater scheme (Baumeister et al., 2013; Garland, Farb, Goldin, \& Fredrickson, 2015; Reker, 2000). The ability to find meaning in negative life experiences is acknowledged to be a powerful coping mechanism and provides a foundation for resilience in the face of obstacles and stressors (Kashdan \& McKnight, 2009; King, Hicks, Krull, \& Del Gaiso, 2006). A strong meaning framework may therefore provide a buffer against the negative effects of interpersonal adversity on suicidal thoughts and behaviors (Heisel \& Flett, 2004; Kleiman \& Beaver, 2013).

In a study of older adults, meaning in life mediated the relationship between high levels of perceived burdensomeness and the prospective prediction of suicidal ideation, suggesting that perceptions of burdensomeness may contribute to suicidality by reducing perceptions of meaning (Van Orden, Bamonti, King, \& Duberstein, 2012). Similarly, the presence of meaning in life mediated the relationship between perceived burdensomeness, thwarted belongingness, and suicidal ideation over an eight-week period (Kleiman \& Beaver, 2013). Moreover, presence of 
meaning at baseline predicted reduced risk for suicidality at follow-up beyond other resilience factors such as gratitude and social support. Thus, interventions targeting an increase in perceptions of meaning may enhance resilience to proximal interpersonal antecedents of suicidal desire.

One way to safely test the value of meaning-making interventions that may increase resilience to the interpersonal adversity thought to causally underlie suicidal desire is to enhance perceptions of meaningfulness experimentally, and to examine the effect on persistence during conditions of experimentally-induced perceived burdensomeness and thwarted belongingness. That is, rather than testing hypotheses that directly involve suicidal thoughts and behaviors, the aim is to manipulate within a controlled environment risk and resilience variables that are relevant to suicide and test their effects on a conceptually and theoretically relevant analogue outcome. Specifically, the ability to persist rather than escape from situations of interpersonal adversity is arguably a key determinant of vulnerability or resilience to the interpersonal factors thought to underlie suicidal desire. Indeed, evidence suggests that the thwarting of fundamental human needs for competence and relatedness has a detrimental impact on goal-directed motivation to persist (Deci \& Ryan, 2000; Ryan \& Deci, 2000), and can lead to self-defeating behaviors, including suicide (Tucker \& Wingate, 2014; Twenge, Catanese, \& Baumeister, 2002; Van Orden et al., 2010). A decline in willingness to persist in the face of experimentallyheightened perceptions of burdensomeness and thwarted belongingness mirrors similar processes as those thought to causally drive the desire to escape such interpersonal adversity via suicide (Joiner, 2005).

In the current study we use the Interpersonal Persistence Task (Collins et al., 2016) to examine the influence of perceived meaningfulness on the desire to escape from interpersonal 
adversity. We manipulate perceptions of meaningfulness by asking participants to select a charitable cause to donate to a small amount of money provided by the experimenter as part of their participation. The aim of this donation is to provide participants with a "silver lining" that may enhance their ability to find meaning even when their experience of the task may be negative (Taylor, 1989). Altruistic behaviors such as charitableness are important sources of meaning across the lifespan by increasing one's sense that life has purpose and value (Baumeister et al., 2013; Hill et al., 2013; Ryan, Huta, \& Deci, 2008). Moreover, altruism is associated with greater resilience, persistence, and adaptation in the face of stress (Kashdan \& McKnight, 2009; Southwick, Vythilingam, \& Charney, 2005). Thus, linking participation in the experiment to a charitable donation may infuse the task with a sense of meaning that protects against deficits in persistence caused by elevated perceived burdensomeness and thwarted belongingness.

The aim was to test whether increasing perceptions of meaningfulness would buffer desire to escape under conditions of heightened perceived burdensomeness and thwarted belongingness (high PB-TB). It was hypothesized that participants in a condition given the opportunity to donate to a charitable cause (high task-extrinsic meaning) would be protected against deficits in persistence compared to participants in a condition not given an opportunity to donate (low task-extrinsic meaning). Given perceptions of meaning are, by definition, personal in nature (Frankl, 1959), participants receiving the task-extrinsic meaning induction would be expected to vary in the extent to which they derive meaning from the charitable donation and the influence this has on their perceptions of meaning during the task. Thus, we expected the buffering effects of the meaning manipulation to vary as a function of perceived meaningfulness during the team task, such that only participants in the high task-extrinsic meaning condition 
who also rated their level of perceived meaningfulness as high would report lower desire to drop out of the task and escape the interpersonal adversity. In contrast, in the low task-extrinsic condition, perceived meaningfulness ratings would be unrelated to task persistence. As the buffering hypothesis proposes that resilience is relevant only when adversity is heightened (Johnson et al., 2011), we expected there to be no relationship between perceived meaningfulness and desire to quit in either high or low task-extrinsic meaning conditions when perceived burdensomeness and thwarted belongingness were not induced (low PB-TB). Finally, because negative affect can reduce persistence at goal-directed activities (Hills, Hill, Mamone, \& Dickerson, 2001), we also accounted for the influence of general psychological distress on the desire to escape.

\section{Method}

\subsection{Participants}

Power analyses for mixed-design analyses of variance (ANOVA) comprising one within subjects factor with six levels (time points) and two between subjects factors (two conditions each with two levels) and for moderated regression analyses with eight predictors (including the interaction term) were conducted using G*Power (Faul, Erdfelder, Lang, \& Buchner, 2007). For the ANOVA, based on a power of 0.80 , an alpha level of .05 , and a medium effect size $(f=$ $0.25)$, an estimated sample size of 76 was required to reliably detect significant main and interaction effects. Similarly, for the moderated regression with a power of 0.80 , an alpha level of .05 , and a medium effect size $\left(f^{2}=0.15\right)$, the required sample size to reliably detect a significant interaction effect split across low and high PB-TB conditions was estimated at 43. Therefore, the current sample provided sufficient statistical power for both sets of analyses. 
Ninety-three undergraduate students $\left(M_{a g e}=19.45\right.$ years, $S D=5.47,66$ females $)$ were recruited based on scores on a screening questionnaire assessing attitudes and behaviors towards charitable causes (Webb, Green, \& Brashear, 2000). Those scoring in the upper $40 \%$ of the distribution, indicating a positive view of charitable causes and that helping others was important to them, were invited. Rather than using a median split, we limited selection to those participants clearly in the top half of the distribution (i.e., top 40\%) in order to maximize the likelihood that they would be susceptible to a meaning-making induction based on the opportunity to donate to a charitable organization. To personalise the available charity options, participants had to have endorsed at least one of six charity organisations on the screening measure, which were the same six options available during the experiment.

Participants were randomly allocated to one of four experimental conditions: (1) low perceived burdensomeness-thwarted belongingness + low task-extrinsic meaning (low PBTB/low task-extrinsic meaning); (2) high perceived burdensomeness-thwarted belongingness + low task-extrinsic meaning (high PB-TB/low task-extrinsic meaning); (3) low perceived burdensomeness-thwarted belongingness + high task-extrinsic meaning (low PB-TB/high taskextrinsic meaning); or (4) high perceived burdensomeness-thwarted belongingness + high taskextrinsic meaning (high PB-TB/high task-extrinsic meaning). All procedures were approved by the University’s Human Research Ethics Committee.

\subsection{Experimental Task and Procedure}

We used an adapted version of the Interpersonal Persistence Task (Collins et al., 2016), a three-player reaction-time task where participants aim to beat a target score by judging whether two characters appearing on screen are alike or different. One point is awarded for a fast and accurate judgment, and one point is deducted for a slow or inaccurate judgment. Points won and 
lost contribute to the participant's individual score and also the team's total score. The task consists of six time blocks divided into three rounds of five trials. After each round, a summary table is displayed showing individual scores, the team total score, and the current target score. While participants are led to believe they are playing with two fellow students, in reality their "co-players" are computer-controlled and points won and lost are manipulated according to odds ratios.

2.21 Burdensomeness and Belongingness Manipulations. To manipulate perceived burdensomeness, participants receive predetermined performance feedback. Participants in the high PB-TB condition are told they are scoring substantially lower than their teammates, while participants in the low PB-TB condition are told they are scoring equal to or better than their teammates. Further, the team total score in the high PB-TB condition is always lower than the team target score, thereby suggesting that the participant's subpar performance is preventing the team from reaching the goal. Belongingness is manipulated using interpersonal feedback statements. After each time interval, participants provide feedback to, and receive feedback from, their teammates via electronic messaging. Participants are unaware that the feedback they receive is computer-generated. In the high $\mathrm{PB}-\mathrm{TB}$ condition participants receive critical feedback, whereas in the low PB-TB condition participants receive supportive feedback.

2.22 Participant Ratings. After receiving the co-player comments, participants rate the extent to which: (1) they feel a burden on their fellow team members (perceived burdensomeness); (2) the other players make them feel part of the team (belongingness); (3) they feel their participation within the task is meaningful (perceived meaning); and (4) if they had a choice, they would rather drop out of the task (desire to quit) on a scale from 0 (not at all true for me) to 6 (very true for me). Relaxation/distress is also rated on a scale from 0 (relaxed) to 6 
(distressed). At the end of the task, participants rate the extent to which they found the task interesting and made an effort to do well on a scale from 0 (not at all true for me) to 6 (very true for me).

2.23 Meaning Manipulation. Participants in the high task-extrinsic meaning condition were told that a proportion of a $\$ 500$ donation would be made on their behalf to a charity selected from a pool of six recognizable and reputable charities reflecting a wide range of causes (e.g., social welfare, animal welfare, environmental protection). To increase the salience of the manipulation (cf., Genevsky, Västfjäll, Slovic, \& Knutson, 2013), a board in the testing room displayed information sheets for each of the six charities consisting of an emotive image and a short mission statement. Participants were also asked to reflect on the reason(s) why contributing to their chosen charity held personal significance for them and to record this on the information sheet. During the Interpersonal Persistence Task, participants received on-screen reminder messages of their charitable donation after receiving co-player feedback (i.e., "Remember, a donation will be made on your behalf to your chosen charity at the end of the experiment"). Participants in the low task-extrinsic meaning condition were not given an opportunity to donate to a charity and received no reminder messages during the task.

\subsection{Questionnaires}

2.31 Charitable Behavior Questionnaire (CBQ; Webb, Green, \& Brashear, 2000). The 9-item CBQ measures attitudes towards helping behavior (e.g., "Helping troubled people with their problems is very important to me") and charitable organizations (e.g., "My image of charitable organizations is positive"). Participants respond on a scale from 1 (strongly disagree) to 5 (strongly agree), with higher scores indicating more positive attitudes towards helping others and charitable organizations. The CBQ has good psychometric properties (Webb et al., 
2000) and internal consistency in the current application was acceptable for both the helping behaviors $(\alpha=.88)$ and attitudes toward charitable organizations $(\alpha=.75)$ subscales.

2.32 Need to Belong to the Team. To establish baseline desire to belong to the team, three items were adapted from an existing scale with sound psychometric properties (Leary, Kelly, Cottrell, \& Schreindorfer, 2013). Participants indicated their level of agreement on a scale from 1 (strongly disagree) to 5 (strongly agree), to the following statements: "When participating in the upcoming team activities in this experiment it is important to me that: (1) others accept me as a person; (2) others care about me as person; and (3) I feel as though I belong." Higher scores indicate greater desire to belong to the team. Internal consistency in the current application was high $(\alpha=.85)$.

2.33 Meaning in Life Questionnaire (MLQ; Steger, Frazier, Oishi, \& Kaler, 2006). The 10-item MLQ consists of two subscales that measure the presence of, and the search for, meaning in life. The current study utilized only the presence subscale (e.g., "I understand my life's meaning"), as this factor has been more consistently associated with suicide resilience in prior research (Kleiman \& Beaver, 2013). Responses range from 1 (this statement is absolutely untrue) to 7 (this statement is absolutely true), with higher scores indicating greater meaning. The MLQ has demonstrated sound psychometric properties in multiple prior studies, including convergent and discriminant validity (Steger et al., 2006; Steger \& Kashdan, 2007), and internal consistency reliability in the current application was high $(\alpha=.89)$.

\subsection{Interpersonal Needs Questionnaire (INQ; Van Orden, Cukrowicz, Witte, \&}

Joiner, 2012). The 15-item INQ assesses perceived burdensomeness (6 items; e.g., "I think I make things worse for the people in my life") and thwarted belongingness ( 9 items; e.g., "I feel disconnected from other people") on a scale from 0 (not at all true of me) to 6 (very true of me), 
with higher scores indicating greater perceptions of burdensomeness and thwarted belongingness. The INQ has demonstrated good validity and reliability in prior studies (Gutierrez et al., 2016; Van Orden, Cukrowicz, et al., 2012) and internal consistency estimates in the current application were excellent for both burdensomeness $(\alpha=.91)$ and belongingness $(\alpha=.90)$ subscales.

2.35 Kessler Psychological Distress Scale (K10; Kessler et al., 2002). The 10-item K10 measures symptoms of psychological distress in the last four weeks (e.g., "About how often did you feel depressed") on a scale from 1 (none of the time) to 5 (all of the time). Scores range from 10 to 50 with higher scores indicating higher distress. Normative bands for K10 scores in Australia are: low distress (10-15), moderate distress (16-21), high distress (22-29), and very high distress (30-50; Cvetkovski, Reavley, \& Jorm, 2012). The K10 has excellent psychometric properties in large representative samples (Cvetkovski et al., 2012; Kessler et al., 2002) and internal consistency reliability in the current application was high $(\alpha=.89)$.

2.36 Suicidal Behavior. Past suicidal behavior was measured using two items from the Self-Injurious Thoughts and Behaviors Interview (SITBI; Nock, Holmberg, Photos, \& Michel, 2007): "How many times in the last year have you thought about suicide?" with answers ranging from 1 (never) to 6 (almost every day); and "How many times in your lifetime have you made an actual attempt to kill yourself in which you had at least some intent to die?" with answers ranging from 0 (never) to 5 (five or more times). The SITBI has demonstrated good psychometric properties, including when administered in self-report format (Latimer, Meade, \& Tennant, 2013). 


\section{$2.4 \quad$ Procedure}

To increase the plausibility of the manipulation, two participants (or a participant and a confederate of the researcher) completed the experiment concurrently in adjacent testing booths and were told there was a third player in the laboratory down the hall. After initial briefing, participants provided informed consent and completed the Need to Belong Measure. During the initial briefing, participants allocated to the high task-extrinsic meaning condition reviewed an information board and chose one charity from six options. They then briefly reflected on why the charity held personal significance for them and recorded this on the charity information sheet. The charity information sheet was then displayed beside the computer throughout the experiment as a visual reminder of the meaningful outcome.

Following the main task, participants completed the questionnaires via online software. A suspicion probe was then administered, consisting of two open-ended questions ("Do you have any comments about the experiment?" and "Do you have any comments about your teammates?") designed to identify participants who may have guessed the nature of the study design. Participants were then debriefed and given contact details of support services.

\section{Results}

\subsection{Data Screening and Participant Characteristics}

Data were normally distributed and no univariate outliers were identified. Two cases were identified as multivariate outliers, however the exclusion of these participants did not alter the outcomes of any subsequent analyses. Descriptive statistics for the questionnaire data are displayed in Table 1. The Need to Belong score was high, suggesting a strong baseline desire to belong to the team during the subsequent team task. Consistent with other undergraduate samples (Collins et al., 2016, 2018), K10 scores indicated moderate levels of psychological distress. 
Thirty-five individuals (37.6\%) had thought about suicide at least once in the past year and eight $(8.6 \%)$ had made at least one suicide attempt in their lifetime.

Table 1

Sample Descriptive Statistics

\begin{tabular}{lcc}
\hline \multicolumn{1}{c}{ Variable } & $M$ & $S D$ \\
\hline Need to belong & 4.02 & 0.80 \\
Presence of meaning in life & 4.79 & 1.25 \\
Perceived burdensomeness & 0.64 & 0.86 \\
Thwarted belongingness & 1.13 & 0.99 \\
Psychological distress & 20.29 & 6.08 \\
\hline
\end{tabular}

\subsection{Manipulation Checks}

Nine participants whose responses on the suspicion probe suggested that they may have guessed the nature of the study design were excluded from subsequent analyses. Mixed-design ANOVA were conducted to check that the experimental manipulation had the intended effects on burdensomeness and belongingness ratings.

3.21 Perceived burdensomeness. A $2 \times 2 \times 6$ mixed-design ANOVA examined the effects of condition (high/low PB-TB) and meaning manipulations (high/low task-extrinsic meaning) across time (blocks 1-6) on perceived burdensomeness. There was a significant main effect of condition, $F(1,80)=314.60, p<.001, \eta_{\text {partial }}^{2}=.78$, confirming that the PB-TB manipulation successfully induced perceived burdensomeness (Figure 1A). There was also a Condition $\times$ Time interaction, $F(3.89,311.08)=7.86, p<.001, \eta^{2}$ partial $=.09$. In the high PB-TB condition, burdensomeness ratings were higher and increased over time, $F(3.51,150.82)=4.52$, $p=.003, \eta_{\text {partial }}^{2}=.10$, whereas in the low PB-TB condition, burdensomeness ratings were lower 
and decreased over time, $F(2.91,96.67)=5.26, p=.003, \eta^{2}$ partial $=.12$. There were no significant effects of task-extrinsic meaning manipulation $(p$ 's $>.05)$.

A)

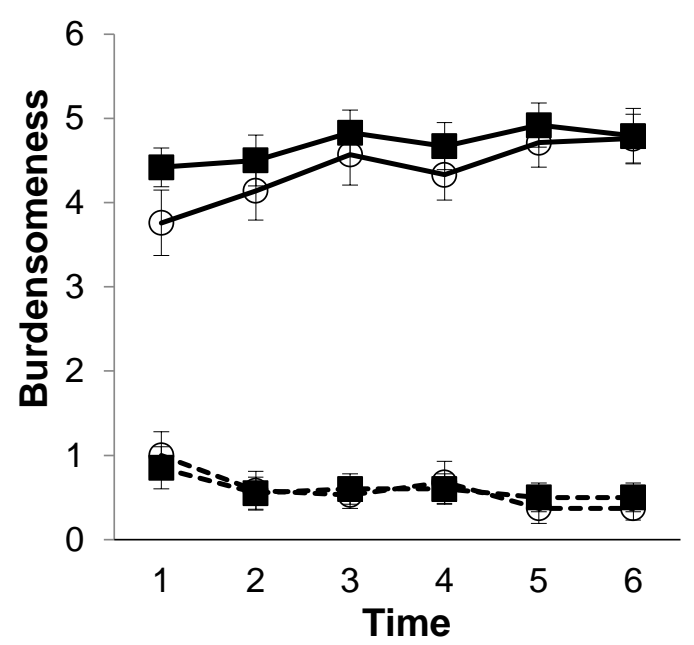

B)

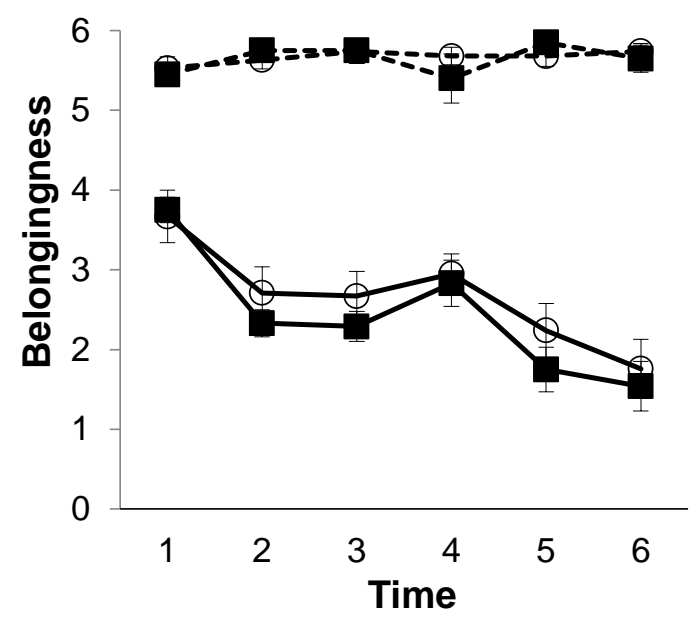

--ם-- Low Meaning / Low PB-TB

-- $\ominus--$ High Meaning / Low PB-TB

$\longrightarrow$ Low Meaning / High PB-TB

- High Meaing / High PB-TB --a-- Low Meaning / Low PB-TB

-- $\Theta--$ High Meaning / Low PB-TB

$\longrightarrow$ Low Meaning / High PB-TB

- High Meaing / High PB-TB

Figure 1. Interpersonal Persistence Task ratings. Panel A shows mean burdensomeness and Panel B shows mean belongingness. Error bars represent standard error of the mean.

3.22 Thwarted belongingness. A $2 \times 2 \times 6$ mixed-design ANOVA examined the effects of condition (high/low PB-TB) and meaning manipulations (high/low task-extrinsic meaning) 
across time (blocks 1-6) on belongingness ratings. A significant main effect of condition, $F(1$, $80)=289.52, p<.001, \eta^{2}$ partial $=.78$, confirmed that the PB-TB manipulation successfully diminished belongingness as intended (Figure 1B). There was also a significant Condition $\times$ Time interaction, $F(3.44,275.33)=24.78, p<.001, \eta^{2}$ partial $=.24$. Figure $1 \mathrm{~B}$ shows that belongingness ratings significantly decreased over time in the high PB-TB condition, $F(3.19$, 137.20) $=28.83, p<.001, \eta_{\text {partial }}^{2}=.40$, but remained consistently high in the low PB-TB condition, $F(2.79,93.28)=1.81, p=.153, \eta^{2}$ partial $=.05$. There were no significant effects of task-extrinsic meaning manipulation ( $p$ 's $>.05)$.

\subsection{Perceived Meaning as a Moderator of Persistence Under Conditions of Interpersonal Adversity and High Task-Extrinsic Meaning}

Descriptive statistics for perceived meaningfulness and task persistence are displayed in Table 2. A $2 \times 2 \times 6$ mixed-design ANOVA examined the effects of condition (high/low PB-TB) and meaning manipulations (high/low task-extrinsic meaning) across time (blocks 1-6) on the desire to quit. There were significant main effects of PB-TB condition, $F(1,82)=53.23, p<$ $.001, \eta_{\text {partial }}^{2}=.39$, and time, $F(3.32,271.95)=14.78, p<.001, \eta^{2}$ partial $=.15$, which were qualified by a significant Condition $\times$ Time interaction, $F(3.32,271.95)=22.12, p<.001, \eta_{\text {partial }}^{2}=.21$. Desire to quit was significantly greater and became stronger over time in the high PB-TB condition, $F(3.47,152.63)=24.99, p<.001, \eta_{\text {partial }}^{2}=.36$, but remained consistently low in the low PB-TB condition, $F(2.49,94.80)=1.68, p=.185, \eta^{2}$ partial $=.04$. 
Table 2

Perceived Meaningfulness and Persistence (Desire to Quit) Ratings Averaged Across the Six Time Blocks by PB-TB Condition and Task-Extrinsic Meaning Condition

\begin{tabular}{lcccccc}
\hline & \multicolumn{2}{c}{ Low PB-TB } & & \multicolumn{2}{c}{ High PB-TB } \\
\cline { 2 - 3 } \cline { 6 - 7 } & Low & High & & Low & High \\
& $M(S D)$ & $M(S D)$ & & $M(S D)$ & $M(S D)$ \\
\hline Peaning & & Meaning & & Meaning & Meaning \\
Derceived Meaningfulness & $4.86(1.25)$ & $5.28(0.85)$ & & $2.40(0.93)$ & $2.38(1.34)$ \\
Desire to Quit & $0.66(1.07)$ & $0.41(0.80)$ & & $2.60(1.58)$ & $3.23(2.04)$ \\
\hline
\end{tabular}

Note. $\mathrm{PB}=$ Perceived Burdensomeness $; \mathrm{TB}=$ Thwarted Belongingness

To test our primary hypothesis that manipulated perceptions of meaning would confer resilience against experimentally-induced perceived burdensomeness and thwarted belongingness, we examined the relationship between perceived meaningfulness ratings and desire to quit (persistence) as a function of high/low meaning condition using the PROCESS macro for SPSS specifying 5,000 bias-corrected 95\% confidence interval bootstrap samples (Hayes, 2013). The analysis was first isolated within the high PB-TB/low task-extrinsic meaning and high $\mathrm{PB}-\mathrm{TB} / \mathrm{high}$ task-extrinsic meaning conditions (i.e., where the interpersonal adversity was experimentally induced). Average perceived meaningfulness was the independent variable, low/high task-extrinsic meaning condition (dummy coded: low meaning condition $=-1$, high meaning condition $=1$ ) was the moderator variable, and mean desire to quit was the outcome variable. Mean interest, effort, distress, burdensomeness, and belongingness ratings were entered as covariates. The overall model explained $63.62 \%$ of the variance in persistence, $F(8,36)=$ $8.38, p<.001$, with meaning condition, effort, and burdensomeness significant unique predictors (Table 3). The meaningfulness rating by high/low task-extrinsic meaning condition interaction term explained an additional $8.78 \%$ of the variance in persistence, $F(1,36)=6.87, p=.013$. This 
interaction is depicted in Figure 2A. Simple slope analyses confirmed that meaningfulness ratings were a strong positive predictor of task persistence in the high task-extrinsic meaning condition $\left(R_{\text {Linear }}^{2}=.54, t=-3.21, p=.003\right)$ but not in the low task-extrinsic meaning condition $\left(R_{\text {Linear }}^{2}=.02, t=-.12, p=.91\right)$.

A) High PB-TB Condition

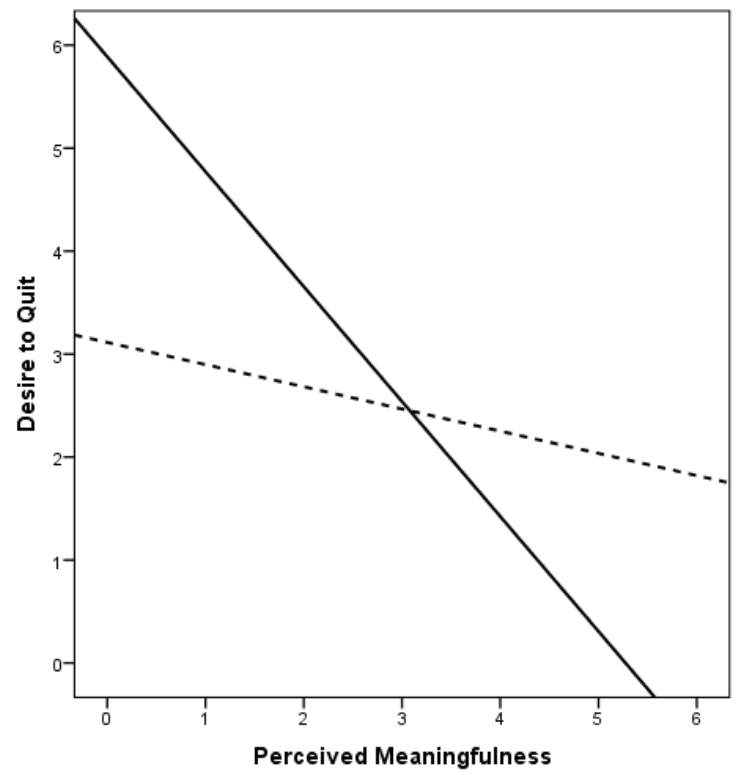

B) Low PB-TB Condition

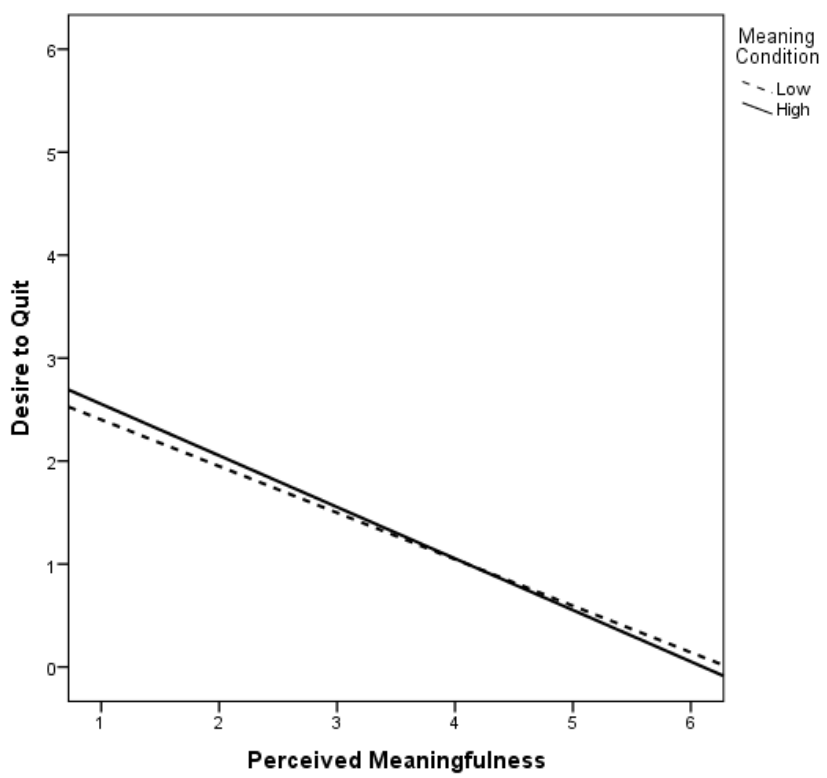

Figure 2. Persistence (desire to quit) as a function of perceived meaningfulness ratings and high/low taskextrinsic meaning conditions. The lines are regression slopes for the simple effects. Panel A shows that under conditions of heightened interpersonal adversity (high PB-TB), higher perceived meaningfulness predicts lower desire to quit in the high, but not low, task-extrinsic meaning condition. Panel B shows that when interpersonal adversity is absent (low PB-TB), there is no relationship between perceived meaningfulness and desire to quit in either high or low task-extrinsic meaning conditions.

Repeating the analysis in the low PB-TB/low task-extrinsic meaning and low PBTB/high task-extrinsic meaning conditions (i.e., where no interpersonal adversity was experimentally induced) showed there was no effect of meaning manipulation condition and the 
meaningfulness rating by high/low task-extrinsic meaning condition interaction term was nonsignificant, $F(1,30)=.05, p=.83$ (see Table 3 and Figure 2B). Thus, when interpersonal adversity was low, there was no relationship between perceived meaningfulness and desire to quit in either high or low task-extrinsic meaning conditions.

Table 3

Moderated Regression Analyses Predicting Persistence (Desire to Quit) Across High and Low PB-TB Conditions

\section{High PB-TB}

\begin{tabular}{|c|c|c|c|c|}
\hline Predictor & $b$ & SE $b$ & $t$ & $p$ \\
\hline Interest & 0.19 & 0.18 & 1.08 & .288 \\
\hline Effort & -0.59 & 0.14 & -4.23 & .002 \\
\hline Distress & -0.01 & 0.19 & -0.01 & .989 \\
\hline Burdensomeness & 0.76 & 0.22 & 3.37 & .002 \\
\hline Belongingness & 0.16 & 0.35 & 0.48 & .641 \\
\hline Meaning Condition & 1.70 & 0.53 & 3.23 & .003 \\
\hline Meaningfulness Ratings & -0.60 & 0.30 & -2.01 & .052 \\
\hline Meaning Condition x Meaningfulness Ratings & -0.56 & 0.21 & -2.62 & .013 \\
\hline \multicolumn{5}{|c|}{ Low PB-TB } \\
\hline Predictor & $b$ & SE $b$ & $t$ & $p$ \\
\hline Interest & -0.11 & 0.20 & -0.54 & .590 \\
\hline Effort & -0.32 & 0.37 & -0.86 & .396 \\
\hline Distress & 0.28 & 0.19 & 1.47 & .152 \\
\hline Burdensomeness & 0.24 & 0.29 & 0.83 & .413 \\
\hline Belongingness & 0.12 & 0.68 & 0.17 & .866 \\
\hline Meaning Condition & -0.02 & 0.12 & -0.16 & .870 \\
\hline Meaningfulness Ratings & -0.21 & 0.34 & -0.62 & .538 \\
\hline Meaning Condition x Meaningfulness Ratings & 0.03 & 0.20 & 0.14 & .890 \\
\hline
\end{tabular}

Note: $\mathrm{PB}=$ Perceived Burdensomeness; $\mathrm{TB}=$ Thwarted belongingness 


\section{Discussion}

The aim of the current study was to examine whether manipulated perceptions of meaningfulness can enhance resilience to perceived burdensomeness and thwarted belongingness. While this approach does not directly test hypotheses concerning suicidal thoughts and behaviors, it does enable the manipulation and testing under controlled conditions of variables that are posited to be causal and proximal antecedents to suicidal desire. As predicted, participants in the high task-extrinsic meaning condition who rated higher levels of perceived meaningfulness displayed greater persistence in the face of heightened perceived burdensomeness and thwarted belongingness. Conversely, in the low task-extrinsic meaning condition where participants were not given the opportunity to contribute to a charity and in the conditions where this interpersonal adversity was not induced, perceived meaningfulness was unrelated to task persistence. The moderating effects of task-extrinsic meaning explained unique variance in desire to quit while controlling for the effects of perceived burdensomeness, thwarted belongingness, and general psychological distress. These findings are consistent with previous evidence linking meaning with greater persistence, adaptation, and recovery in the face of stress (Kashdan \& McKnight, 2009; Park \& George, 2013; Ryan et al., 2008; Southwick et al., 2005), and importantly, longitudinal research showing meaning in life predicts lower suicidal ideation over an eight week period above and beyond the ITS risk factors (Kleiman \& Beaver, 2013). The current experimental evidence complements these previous longitudinal findings by showing manipulated perceptions of meaning confer protection against the deleterious effects of interpersonal adversity on willingness to persist, the erosion of which is a potential antecedent to suicide risk. 
The current findings reinforce the notion that life-sustaining factors play an important role in buffering the effects of interpersonal antecedents to suicidal desire. Contemporary theories of suicide, including the ITS, should therefore consider the role of life-sustaining factors in order to provide a more comprehensive account of suicidal behavior. Such a model might include factors such as mindfulness, perceived meaning, and zest for life situated along a common pathway to suicide resilience. Attending to the present moment with openness and acceptance can increase one's ability to find meaning in adversity and to pursue valued goals with grit (c.f., Duckworth, Peterson, Matthews, \& Kelly, 2007; Garland et al., 2015). Striving towards personal goals may in turn increase vitality and engagement with life as one perceives one has a reason for living (Frankl, 1988). This enhanced engagement with and zest for life might provide an important counterbalance to the death-promoting effects of interpersonal adversity and in doing so confer resilience to suicidality (Collins et al., 2018). In a clinical context, existing evidence-based treatments such as Behavioral Activation (Jacobson, Martell, \& Dimidjian, 2001) and Acceptance and Commitment Therapy (ACT; Hayes, Strosahl, \& Wilson, 2011) already focus on enhancing engagement with life by increasing access to sources of positive reinforcement in the environment and encouraging values-guided action. These approaches provide a ready-made template for clinicians seeking to enhance suicide resilience in the form of meaningful engagement with life.

The current study had some limitations that suggest directions for future research. First, it is important to note that the meaning intervention was successful in buffering the effects of interpersonal adversity only to the extent that the charity donations were perceived as meaningful and relevant to participation in the goal-directed activity being undertaken. The literature suggests that what an individual considers meaningful is deeply personal (Brandstätter, 
Baumann, Borasio, \& Fegg, 2012; Frankl, 1959) and thus, although we attempted to personalize the induction, imposing a single source of meaning (i.e., a contribution to one of six pre-selected charities) in the current experimental context was apparently insufficient to enhance perceptions of meaning in some individuals. This would be consistent with literature showing that when extrinsic motivators are not freely chosen or lack personal significance, the fundamental need for autonomy is thwarted, leading to diminished motivation in goal-directed activities (Deci \& Ryan, 2000). Further, while in the current context the meaning manipulation focused on altruistic behavior, this type of intervention might not have the same strength as other activities such as volunteering or having a career that one is passionate about. These findings highlight the importance of tailoring meaning-making interventions to the individual to ensure goals and activities are chosen and pursued with autonomy. Future studies might allow participants to select a personal reason for participating in the task and in doing so potentially increase the effectiveness of the intervention (c.f., Branstetter-Rost, Cushing, \& Douleh, 2009). A second potential limitation was the selection of participants who viewed contribution to charitable causes as positive and important. While this approach was adopted in order to increase the likelihood that selected participants would be susceptible to the meaning manipulation, it is also possible that individuals with these pre-existing characteristics are already more resilient to perceived burdensomeness and thwarted belongingness. A third limitation is that the dual induction of perceived burdensomeness and thwarted belongingness in the current application means the independent effects of these constructs on task persistence cannot be determined. Although the ITS proposes that it is the joint presence of the interpersonal factors which drives suicidal desire, recent reviews point to a more complex picture where perceived burdensomeness may exert a stronger effect on suicidal desire compared to thwarted belongingness (Chu et al., 
2017; Ma, Batterham, Calear, \& Han, 2016) but thwarted belongingness may be more relevant as a predictor of suicidal behavior in the context of acquired capability for suicide (Chu et al., 2017). Future research could independently manipulate the ITS risk factors in order to tease apart their independent and interactive effects on desire to escape.

In conclusion, the current study found that manipulated perceptions of meaningfulness confer protection against desire to escape in the face of heightened perceived burdensomeness and thwarted belongingness. This suggests that interventions designed to increase one's sense that life has meaning and purpose could make a valuable contribution to suicide prevention. The current findings also highlight that contemporary theories of suicide should account for the role of life-sustaining factors such as perceived meaning in order to increase the specificity of their causal predictions. 
Funding: This research did not receive any specific grant from funding agencies in the public, commercial, or not-for-profit sectors

\section{Declarations of interest: None}




\section{References}

Barzilay, S., Feldman, D., Snir, A., Apter, A., Carli, V., Hoven, C. W., ... Wasserman, D. (2015). The interpersonal theory of suicide and adolescent suicidal behavior. Journal of Affective Disorders, 183, 68-74. https://doi.org/10.1016/j.jad.2015.04.047

Baumeister, R. F. (1990). Suicide as escape from self. Psychological Review, 97(1), 90-113. https://doi.org/10.1037/0033-295X.97.1.90

Baumeister, R. F., Vohs, K. D., Aaker, J. L., \& Garbinsky, E. N. (2013). Some key differences between a happy life and a meaningful life. The Journal of Positive Psychology, 8(6), 505-516. https://doi.org/10.1080/17439760.2013.830764

Brandstätter, M., Baumann, U., Borasio, G. D., \& Fegg, M. J. (2012). Systematic review of meaning in life assessment instruments. Psycho-Oncology, 21(10), 1034-1052. https://doi.org/10.1002/pon.2113

Branstetter-Rost, A., Cushing, C., \& Douleh, T. (2009). Personal Values and Pain Tolerance: Does a Values Intervention Add to Acceptance? The Journal of Pain, 10(8), 887-892. https://doi.org/10.1016/j.jpain.2009.01.001

Cheavens, J. S., Cukrowicz, K. C., Hansen, R., \& Mitchell, S. M. (2016). Incorporating resilience factors into the interpersonal theory of suicide: The role of hope and self-forgiveness in an older adult sample. Journal of Clinical Psychology, 72(1), 58-69. https://doi.org/10.1002/jclp.22230

Christensen, H., Batterham, P. J., Soubelet, A., \& Mackinnon, A. J. (2013). A test of the Interpersonal Theory of Suicide in a large community-based cohort. Journal of Affective Disorders, 144(3), 225-234. https://doi.org/10.1016/j.jad.2012.07.002

Chu, C., Buchman-Schmitt, J. M., Stanley, I. H., Hom, M. A., Tucker, R. P., Hagan, C. R., ... Joiner, T. E. (2017). The interpersonal theory of suicide: A systematic review and meta-analysis of a decade of cross-national research. Psychological Bulletin, 143(12), 1313-1345. https://doi.org/10.1037/bul0000123 
Collins, K. R. L., Best, I., Stritzke, W. G. K., \& Page, A. C. (2016). Mindfulness and zest for life buffer the negative effects of experimentally-induced perceived burdensomeness and thwarted belongingness: Implications for theories of suicide. Journal of Abnormal Psychology, 125(5), 704-714. https://doi.org/10.1037/abn0000167

Collins, K. R. L., Stritzke, W. G. K., Page, A. C., Brown, J. D., \& Wylde, T. J. (2018). Mind full of life: Does mindfulness confer resilience to suicide by increasing zest for life? Journal of Affective Disorders, 226, 100-107. https://doi.org/10.1016/j.jad.2017.09.043

Cvetkovski, S., Reavley, N. J., \& Jorm, A. F. (2012). The prevalence and correlates of psychological distress in Australian tertiary students compared to their community peers. Australian and New Zealand Journal of Psychiatry, 46(5), 457-467. https://doi.org/10.1177/0004867411435290

Deci, E. L., \& Ryan, R. M. (2000). The "what" and "why” of goal pursuits: Human needs and the selfdetermination of behavior. Psychological Inquiry, 11(4), 227-268. https://doi.org/10.1207/S15327965PLI1104_01

Duckworth, A. L., Peterson, C., Matthews, M. D., \& Kelly, D. R. (2007). Grit: Perseverance and passion for long-term goals. Journal of Personality and Social Psychology, 92(6), 1087-1101. https://doi.org/10.1037/0022-3514.92.6.1087

Faul, F., Erdfelder, E., Lang, A.-G., \& Buchner, A. (2007). G*Power 3: A flexible statistical power analysis program for the social, behavioral, and biomedical sciences. Behavior Research Methods, 39(2), 175-191. https://doi.org/10.3758/BF03193146

Frankl, V. E. (1959). Man's search for meaning. Boston, Massachusetts: Beacon Press.

Frankl, V. E. (1988). The will to meaning: Foundations and applications of Logotherapy. New York, NY: Meridian.

Garland, E. L., Farb, N. A. S., Goldin, P. R., \& Fredrickson, B. L. (2015). Mindfulness broadens awareness and builds eudaimonic meaning: A process model of mindful positive emotion regulation. Psychological Inquiry, 26(4), 293-314. https://doi.org/10.1080/1047840X.2015.1064294 
Genevsky, A., Västfjäll, D., Slovic, P., \& Knutson, B. (2013). Neural underpinnings of the identifiable victim effect: Affect shifts preferences for giving. The Journal of Neuroscience, 33(43), 17188. https://doi.org/10.1523/JNEUROSCI.2348-13.2013

George, S. E., Collins, K. R. L., Cao, T., Stritzke, W. G. K., \& Page, A. C. (2017). An experimental test of the interpersonal in the interpersonal theory of suicide. Journal of Experimental Psychopathology, In Press(0), 1-20. https://doi.org/10.5127/jep.060316

Gutierrez, P. M., Pease, J., Matarazzo, B. B., Monteith, L. L., Hernandez, T., \& Osman, A. (2016). Evaluating the psychometric properties of the interpersonal needs questionnaire and the acquired capability for suicide scale in military veterans. Psychological Assessment. https://doi.org/10.1037/pas0000310

Hayes, A. F. (2013). Introduction to Mediation, Moderation, and Conditional Process Analysis: A Regression-Based Approach. New York, NY: Guilford Press.

Hayes, S. C., Strosahl, K. D., \& Wilson, K. G. (2011). Acceptance and commitment therapy, second edition: The process and practice of mindful change. New York, NY: Guilford Press.

Heisel, M. J., \& Flett, G. L. (2004). Purpose in life, satisfaction with life, and suicide ideation in a clinical sample. Journal of Psychopathology and Behavioral Assessment, 26(2), 127-135. https://doi.org/10.1023/B:JOBA.0000013660.22413.e0

Hill, C. E., Bowers, G., Costello, A., England, J., Houston-Ludlam, A., Knowlton, G., .. Thompson, B. J. (2013). What's it all about? A qualitative study of undergraduate students' beliefs about meaning of life. Journal of Humanistic Psychology, 0022167813477733. https://doi.org/10.1177/0022167813477733

Hills, A. M., Hill, S., Mamone, N., \& Dickerson, M. (2001). Induced mood and persistence at gaming. Addiction, 96(11), 1629-1638.

Jacobson, N. S., Martell, C. R., \& Dimidjian, S. (2001). Behavioral Activation Treatment for Depression: Returning to Contextual Roots. Clinical Psychology: Science and Practice, 8(3), 255-270. https://doi.org/10.1093/clipsy.8.3.255 
Johnson, J., Wood, A. M., Gooding, P. A., Taylor, P. J., \& Tarrier, N. (2011). Resilience to suicidality: The buffering hypothesis. Clinical Psychology Review, 31(4), 563-591. https://doi.org/10.1016/j.cpr.2010.12.007

Joiner, T. E. (2005). Why people die by suicide. Cambridge, Massachusetts: Harvard University Press.

Joiner, T. E., Van Orden, K. A., Witte, T. K., Selby, E. A., Ribeiro, J. D., Lewis, R., \& David, M. (2009). Main predictions of the interpersonal-psychological theory of suicidal behavior: Empirical tests in two samples of young adults. Journal of Abnormal Psychology, 118(3), 634-646. https://doi.org/10.1037/a0016500

Kashdan, T. B., \& McKnight, P. E. (2009). Origins of purpose in life: Refining our understanding of a life well lived. Psychological Topics, 18(2), 303-316.

Kessler, R. C., Andrews, G., Colpe, L. J., Hiripi, E., Mroczek, D. K., Normand, S. L. T., ... Zaslavsky, A. M. (2002). Short screening scales to monitor population prevalences and trends in non-specific psychological distress. Psychological Medicine, 32(06), 959-976. https://doi.org/10.1017/S0033291702006074

King, L. A., Hicks, J. A., Krull, J. L., \& Del Gaiso, A. K. (2006). Positive affect and the experience of meaning in life. Journal of Personality and Social Psychology, 90(1), 179-196. https://doi.org/10.1037/0022-3514.90.1.179

Kleiman, E. M., \& Beaver, J. K. (2013). A meaningful life is worth living: Meaning in life as a suicide resiliency factor. Psychiatry Research, 210(3), 934-939. https://doi.org/10.1016/j.psychres.2013.08.002

Latimer, S., Meade, T., \& Tennant, A. (2013). Measuring engagement in deliberate self-harm behaviours: psychometric evaluation of six scales. BMC Psychiatry, 13(4). https://doi.org/10.1186/1471$244 X-13-4$

Leary, M. R., Kelly, K. M., Cottrell, C. A., \& Schreindorfer, L. S. (2013). Construct validity of the need to belong scale: Mapping the nomological network. Journal of Personality Assessment, 95(6), 610-624. https://doi.org/10.1080/00223891.2013.819511 
Ma, J., Batterham, P. J., Calear, A. L., \& Han, J. (2016). A systematic review of the predictions of the Interpersonal-Psychological Theory of Suicidal Behavior. Clinical Psychology Review, 46, 34 45. https://doi.org/10.1016/j.cpr.2016.04.008

Nock, M. K., Holmberg, E. B., Photos, V. I., \& Michel, B. D. (2007). Self-injurious thoughts and behaviors interview: Development, reliability, and validity in an adolescent sample. Psychological Assessment, 19(3), 309-317. https://doi.org/10.1037/1040-3590.19.3.309

O’Connor, R. C. (2011). Toward an integrated motivational - volitional model of suicidal behavior. In R. C. O’Connor, S. Platt, \& J. Gordon (Eds.), International Handbook of Suicide Prevention: Research, Policy and Practice (pp. 181-198). Chichester: Wiley Blackwell.

Park, C. L. (2010). Making sense of the meaning literature: An integrative review of meaning making and its effects on adjustment to stressful life events. Psychological Bulletin, 136(2), 257-301. https://doi.org/10.1037/a0018301

Park, C. L., \& George, L. S. (2013). Assessing meaning and meaning making in the context of stressful life events: Measurement tools and approaches. The Journal of Positive Psychology, 8(6), 483504. https://doi.org/10.1080/17439760.2013.830762

Reker, G. T. (2000). Exploring Existential Meaning: Optimizing Human Development Across the Life Span. (G. T. Reker \& K. Chamberlain, Eds.). Thousand Oaks, CA: SAGE Publications.

Ryan, R. M., \& Deci, E. L. (2000). Self-determination theory and the facilitation of intrinsic motivation, social development, and well-being. American Psychologist, 55(1), 68-78. https://doi.org/10.1037/0003-066X.55.1.68

Ryan, R. M., Huta, V., \& Deci, E. L. (2008). Living well: A self-determination theory perspective on eudaimonia. In The Exploration of Happiness (Vol. 9, pp. 117-139). Dordrecht: Springer International Publishing. Retrieved from http://search.ebscohost.com/login.aspx?direct=true \&db=eoh\&AN=EP28527071\&site=ehost-live 
Southwick, S. M., Vythilingam, M., \& Charney, D. S. (2005). The psychobiology of depression and resilience to stress: Implications for prevention and treatment. Annual Review of Clinical Psychology, 1, 255-291.

Steger, M. F., Frazier, P., Oishi, S., \& Kaler, M. (2006). The meaning in life questionnaire: Assessing the presence of and search for meaning in life. Journal of Counseling Psychology, 53(1), 80-93. https://doi.org/10.1037/0022-0167.53.1.80

Steger, M. F., \& Kashdan, T. B. (2007). Stability and specificity of meaning in life and life satisfaction over one year. Journal of Happiness Studies, 8(2), 161-179. http://dx.doi.org.ezproxy.library.uwa.edu.au/10.1007/s10902-006-9011-8

Taylor, S. E. (1989). Positive illusions: Creative self-deception and the healthy mind (Vol. xv). New York, NY, US: Basic Books.

Tucker, R. P., \& Wingate, L. R. (2014). Basic need satisfaction and suicidal ideation: A selfdetermination perspective on interpersonal suicide risk and suicidal thinking. Archives of Suicide Research, 18(3), 282-294. https://doi.org/10.1080/13811118.2013.824839

Twenge, J. M., Catanese, K. R., \& Baumeister, R. F. (2002). Social exclusion causes self-defeating behavior. Journal of Personality and Social Psychology, 83(3), 606-615. https://doi.org/10.1037/0022-3514.83.3.606

Van Orden, K. A., Bamonti, P. M., King, D. A., \& Duberstein, P. R. (2012). Does perceived burdensomeness erode meaning in life among older adults? Aging \& Mental Health, 16(7), 855860. https://doi.org/10.1080/13607863.2012.657156

Van Orden, K. A., Cukrowicz, K. C., Witte, T. K., \& Joiner, T. E. (2012). Thwarted belongingness and perceived burdensomeness: Construct validity and psychometric properties of the Interpersonal Needs Questionnaire. Psychological Assessment, 24(1), 197-215. https://doi.org/10.1037/a0025358 
Van Orden, K. A., Lynam, M. E., Hollar, D., \& Joiner, T. E. (2006). Perceived burdensomeness as an indicator of suicidal symptoms. Cognitive Therapy and Research, 30(4), 457-467. https://doi.org/10.1007/s10608-006-9057-2

Van Orden, K. A., Witte, T. K., Cukrowicz, K. C., Braithwaite, S. R., Selby, E. A., \& Joiner, T. E. (2010). The interpersonal theory of suicide. Psychological Review, 117(2), 575-600. https://doi.org/10.1037/a0018697

Webb, D. J., Green, C. L., \& Brashear, T. G. (2000). Development and validation of scales to measure attitudes influencing monetary donations to charitable organizations. Journal of the Academy of Marketing Science, 28(2), 299-309. 